\title{
Minimal Upper Mantle Temperature Variations Consistent With Observed Heat Flow and Plate Velocities
}

\author{
William M. Kaula \\ Department of Earth \& Space Sciences, University of Californa, Los Angeles
}

\begin{abstract}
The momentum equations applied to $5^{\circ}$ block means are integrated from the observed surface plate velocities downward to a depth of $280 \mathrm{~km}$, assuming no lateral heterogeneities in density or viscosity. It is assumed that $85 \%$ of the global heat production $Q_{G}=0.85 \times 4.0 \times 10^{13} \mathrm{~W}$ comes from below 280 $\mathrm{km}$ and that at this level the transfer is fully convective. A temperature field $T$ is inferred at depth 280 $\mathrm{km}$ by minimizing the quantity $\int\left|T-T_{0}\right|^{n} d S+n \lambda\left\{Q_{G}-\rho C \int\left(T-T_{0}\right) v, d S\right\}$, where the integrals are over the sphere, $\rho$ is density, $C$ is heat capacity, $v$, is radial velocity, $T_{0}$ is a prescribed mean, and $\lambda$ is a Lagrangian multiplier. Norms $n$, ranging from 1.5 to 2.5 , are tried. The intervening temperature fields are then inferred, integrating the energy equation downward by using the previously calculated velocity field. This integration is subject to the limitations that the derivative of the temperature with respect to depth is everywhere sufficient to attain the fully convecting temperature, but never less than adiabatic. A surface heat flow based on observations plus age and tectonic setting is used. The principal inferences are: (1) the greatest lateral variations in temperature, $\sim 1000^{\circ} \mathrm{C}$, occur with the top $20 \mathrm{~km}$; (2) the greatest advection, $\sim 200^{\circ} \mathrm{C} / \mathrm{m}$. y., occurs within the top $20 \mathrm{~km}$; (3) below $50 \mathrm{~km}$, the greatest departures of temperature from the mean are negative "tongues," reaching an extreme of about $-800^{\circ} \mathrm{C}$ at depth $100 \mathrm{~km}$; (4) below $50 \mathrm{~km}$, heat transfer becomes more convective than conductive; (5) at the fully convecting level, $280 \mathrm{~km}$, temperature variations are at least $\pm 180^{\circ} \mathrm{C}$ about the mean. The principal defect in the entire calculation is unrealistically low temperatures arising from unrepresentatively low surface heat flows. The principal defect of the model probably arises from the assumption that all heat transfer at a depth of $280 \mathrm{~km}$ is representable by $5^{\circ}$ means in velocity and temperature.
\end{abstract}

\section{INTRODUCTION}

The purpose of this paper is to infer upper mantle temperature variations from plate velocities and heat flow data. My intent is to use these temperature distributions as a starting model for interpreting variations of the gravity field. They should also be of interest for interpreting petrological, seismological, and other data.

The guiding principles of the approximate calculation carried out in this paper is that it be as complete as possible, subject to three main limitations: (1) the equations of motion are decoupled from the energy equation; (2) the boundary layer variation in temperature with depth is determined by local conditions; and (3) the resolution is limited to that expressible by $5^{\circ}$ square means (or 36th degree harmonics). Even with these limitations, the work is a considerable elaboration on any similar analyses previously made. A twostage calculation results.

In the first stage, velocities as functions of radius, latitude, and longitude (or alternatively, radius, harmonic degree, and order) are calculated by starting from zero vertical velocity and given horizontal velocities at the surface and using the mass and momentum conservation equations with densities and viscosities prescribed as functions of depth only. In the second stage the temperatures are calculated by starting from uniform temperature and given heat flow at the surface and using the energy equation with the already calculated velocities and prescribed heat sources and thermal properties (densities, heat capacities, and conductivities) at depth. The first-stage calculation is essentially identical to that of Hager and O'Connell [1978, 1979]. The second-stage calcu-

Copyright 1983 by the American Geophysical Union.

Paper number 3B 1054.

0148-0227/83/003B-1054\$05.00 lation has some similarities to those of Chapman and Pollack [1975, 1980], Pollack and Chapman [1977], and Sclater et al . [1980]. It differs, however, in taking more explicit account of the velocity field.

The second stage, as stated above, is insufficient, however, to obtain a temperature distribution approximating that of a convecting regime. A global condition characteristic of this regime must be applied. The principal idea applied in this work is that of a fully convecting level. For such a level $r$, we can write, for the total global heat flow $Q_{G}$ :

$$
\int_{S} \rho C v_{r} T r_{c}^{2} d S \approx \int_{S}\left\{Q a^{2}-\int_{r_{r}}^{a} R r^{2} d r\right\} d S=Q_{G}
$$

where $\rho$ is density, $C$ is heat capacity, $v_{r}$ is radial velocity, $T$ is temperature, $Q$ is surface heat flow, $R$ is the density of intrinsic heat sources, $a$ is surface radius, and the integrals are over the unit sphere. In this studv. $280 \mathrm{~km}$ was chosen as the fully convecting level, as a reasonable compromise. At this depth the only regions of perceptible conductive transfer would be under old continents, which are of slight effect on the global heat budget. To go deeper, however, would risk more error in the velocity field extrapolated from the surface plate motions.

There are three principal objections to the program outlined here: (1) the heat flow is affected by relatively shallow phenomena; (2) the velocity field is affected by lateral variations in lithospheric rheology; and (3) the temperature variation with respect to depth is affected by location with respect to the velocity field.

Oceanic heat flow measurements can be unrepresentatively low because of convection in the shallow permeable layer. An adjustment for these effects near ocean rises is made in the heat flow compilation of Chapman and Pollack [1980], which is the one used. The question is to what extent is adjustment needed well off the rise, perhaps as far as $\mathbf{8 0}$ 
million years [Anderson and Skilbeck, 1981; Embley et al., 1983]. The answer depends on detailed examination of the circumstances of the observations. Meanwhile, it is an open question as to whether some of the low heat flow values to the flanks of ocean rises indicate secondary return flows. On the continents there are marked heat flow variations arising from crustal sources [Sclater et al. 1981] as well as mantlelithosphere interaction. In view thereof I assume a uniform heat flow at the base of the continental crust. Despite the appreciable irregularities of shallow origin, the heat flow must still be a strong constraint on upper mantle temperatures.

Lateral variations in lithospheric rheology, arising mainly from temperature dependence, result in marked lateral variations in stress [Hager and $O^{\prime}$ Connell, 1981] and hence must affect the velocity field. However, in the present calculation the stress field is not of interest per se, while its effects on the velocity field are not manifest until they are integrated over some distance. For this reason, the integrations of Hager and O'Connell [1978, 1979], with only radial variation in viscosity, were successful in reproducing the flow pattern in subduction zones. In the present calculation the main effect of lithospheric rheology is taken into account by starting from the surface velocity field in which nonzero divergence is confined to plate margins. Hence the error is in the second-order variations.

In integrating the energy equation downward by using the $5^{\circ}$ averaged velocity field, provision must be made for contributions to the averaged advection $\mathrm{v} \cdot \nabla T$ from smallerscale variations. This provision necessitates assumptions that are based on boundary layer theory [e.g., Olson and Corcos, 1980], computer experiment [e.g., McKenzie et al., 1974], or more local analyses of data [e.g., Lewis, 1981]. The theoretical and computer models indicate that temperature variation with respect to depth does not increase monotonically to the flanks of a plume, but the detailed data analyses, nonetheless, get a good fit with a monotonic increase. A global-scale model cannot hope to do better; in any case the variation of temperature from a monotonic gradient is small compared to the total temperature change between surface and asthenosphere.

\section{Fundamental Equations}

Under the assumptions stated in the introduction, the conservation equations (7)-(9) in Kaula [1980] reduce to

$$
\begin{gathered}
0=\nabla \cdot(\rho \mathbf{v}) \\
\mathbf{0}=-\nabla \delta p+\partial\left\{\eta \partial v_{r} / \partial r\right\} / \partial r+\eta \nabla_{H}^{2} \mathbf{v} \\
\rho C \partial T / \partial t=\nabla \cdot(K \nabla T)+q_{R}+Z
\end{gathered}
$$

where $\mathbf{v}$ is velocity, $\delta p$ is the variation of pressure from hydrostatic, $\eta$ is viscosity, $K$ is thermal conductivity, $q_{R}$ is the radioactive heat source, and $Z$ is the nonlinear term:

$$
Z=-\rho C \mathbf{v} \cdot\left\{\nabla T-\lambda T_{a}{ }^{\prime}\right\}+\eta[\mathrm{E}: \mathrm{E}] / 2
$$

where $\lambda$ is the radial unit vector, $T_{a}{ }^{\prime}$ is the adiabatic gradient, and $E$ is the strain rate tensor.

\section{MOMENTUM INTEGRATION}

The integration of the poloidal velocity field was first done in terms of spherical harmonics, which results in sets of four first-order differential equations: one set for each harmonic degree and order, as described in Kaula [1975a] and Hager and O'Connell [1978, 1979]. The viscosities used were as given in Kaula [1980].

However, this procedure resulted in some unrealistic oscillations in $v_{r}$ ("Gibbs effects") to the flanks of rapidly spreading rises. These oscillations persisted to some extent, even when the surface harmonic coefficients were obtained by harmonic analysis of divergence along plate margins using $1^{\circ}$ intervals:

$$
v_{s}^{\prime}=\frac{1}{4 \pi L} \int w Y_{l} d B
$$

where the notation is as in Kaula [1975a, 1980]: $v_{s}^{l}$ is the poloidal velocity scalar; $l$ in the indices is an abbreviation for degree and order $l, m ; L$ is $l(l+1) ; w$ is the spreading velocity, positive on a rise; $Y_{l}$ is the spherical harmonic; and the integration is along all plate margins. Equation (6) is obtained by applying the divergence theorem to the usual equation in terms of derivatives [(12) in Kaula, 1975a] and the relationship between the horizontal divergence and scalar coefficients of velocity [Kaula, 1978].

In the end it was found to be more accurate, as well as to be appreciably more economical, to integrate spatially. The equations for the harmonic coefficients [(45) and (47), Kaula, 1975a] were multiplied by $Y_{l}$, the conversion $L Y_{l}=-\nabla_{H}^{2} Y_{l}$ [(10), Kaula, 1975a] applied, and a summation made over $l$ to obtain:

$$
\begin{aligned}
& v_{r}^{\prime}=-\frac{2}{r} v_{r}-\frac{1}{r} D \\
& v_{s}^{\prime}=-\frac{1}{r} v_{r}+\frac{1}{r} v_{s}+\frac{1}{\eta} \sigma_{s} \\
& \sigma_{r}^{\prime}=\frac{12 \eta}{r^{2}} v_{r}+\frac{6 \eta}{r^{2}} D-\frac{1}{r} \nabla_{H}^{2} \sigma_{s} \\
& \sigma_{s}^{\prime}=-\frac{6 \eta}{r^{2}} v_{r}-\frac{2 \eta}{r^{2}}\left(2 D+v_{s}\right)-\frac{1}{r} \sigma_{r}-\frac{3}{r} \sigma_{s} \\
& D^{\prime}=-\frac{1}{r} \nabla_{H}^{2} v_{r}+\frac{1}{r} D+\frac{1}{\eta} \nabla_{H}^{2} \sigma_{s},
\end{aligned}
$$

where the primes denote radial derivatives, $\sigma_{r}$ and $\sigma_{3}$ are the $r r$ and $r s$ components of stress, $D$ is the horizontal divergence of the velocity,

$$
D=\nabla_{H} \cdot \mathrm{v}=\nabla_{H}^{2} v_{\mathrm{s}}
$$

and $\nabla_{H}{ }^{2}$ is the horizontal Laplacian on the unit sphere:

$$
\nabla_{H}^{2}=\frac{\partial^{2}}{\partial \theta^{2}}+\cot \theta \frac{\partial}{\partial \theta}+\frac{1}{\sin ^{2} \theta} \cdot \frac{\partial^{2}}{\partial \phi^{2}}
$$

where $\theta$ is co-latitude and $\phi$ is longitude. The fifth equation for $D$ may seem redundant to the second; however, it preserves the accurately known information of the surface horizontal divergence.

Equation (7) was applied to $5^{\circ}$ square means, starting from the surface with $v_{r}, \sigma_{r}, \sigma_{s}$ zero and $v_{s}, D$ at the observed values. In the horizontal direction, the Laplacians $\nabla_{H}{ }^{2} v$, and $\nabla_{H}{ }^{2} v_{s}$ were obtained by Fourier analysis along meridi- 
ans and parallels to half the Nyquist frequency, $l=18$. In the radial direction the step for Runge-Kutta integration, determined mainly by the difficulties of the energy equation described below, was $10-\mathrm{km}$ to $100-\mathrm{km}$ depth and then increased in stages to $40 \mathrm{~km}$ for the last three steps to the assumed fully convecting level at $280-\mathrm{km}$ depth. The greatest radial velocities attained were $+100 \mathrm{~mm} / \mathrm{yr}$ under the southeast Pacific rise and $-100 \mathrm{~mm} / \mathrm{yr}$ in the Japan subduction zone.

\section{ENERGY INTEGRATION}

The integration of the energy is more complicated because, for any feasible global representation, there are significant contributions to the nonlinear term $Z$ from above the truncation level. Hence any integration must allow for these contributions, regardless of whether harmonic coefficients or area means are employed. For clarity the discussion will be in terms of area means for depth $z$ in a half space; the actual calculations took sphericity into account. Consideration was given to putting the mathematics in an appendix. However, the mathematics is so intertwined with physical reasonings that such an arrangement appears to be unfeasible.

Define sources $S$

$$
S=q_{R}+Z-\rho C_{v} \partial T / \partial t
$$

and heat flow $Q$ as

$$
\mathrm{Q}=-\mathrm{K} \partial \mathrm{T} / \partial \mathrm{r}=\mathrm{K} \partial \mathrm{T} / \partial \mathrm{z}
$$

To integrate from a depth $z_{1-1}$ down to a depth $z_{1}, z_{1}>z_{1-1}$, requires knowledge of the effective sources over the range from $z_{i-1}$ to $z_{1}$. If depth $z_{1}$ is close enough to depth $z_{1-1}$ that sources $S$ and inverse conductivity $1 / K$ can be assumed to vary linearly between them, then for the variables at depth $z_{1}:$

$$
Q_{1}=Q_{i-1}-\left(S_{i-1}+S_{i}\right) \Delta z / 2
$$

and

$$
T_{i}=T_{i-1}+B_{i} Q_{\imath-1}-G_{i} S_{i-1}-A_{i} S_{l}
$$

where

$$
\begin{aligned}
& B_{i}=\left(1 / K_{t-1}+1 / K_{t}\right) \Delta z / 2 \\
& G_{i}=\left(5 / K_{t-1}+3 / K_{l}\right)(\Delta z)^{2} / 24 \\
& \hat{A}_{i}=\left(1 / K_{t-1} \quad 3 / \boldsymbol{X}_{l}\right)(\Delta z)^{2 / 24}
\end{aligned}
$$

Normally sources $S_{i-1}$ will be given from a previous integration step (starting with $S_{0}=q_{R}$ at the surface). The problem is to estimate $S_{i}$. If dissipation $E: E$ in (5) is neglected, then

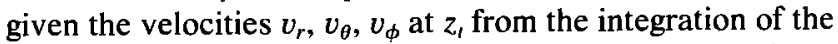
momentum equations, an estimate of temperature gradients at depth $z_{l}$ is needed to calculate an advection. Define an extrapolated temperature $\hat{T}$, by assuming $S_{i}=S_{t-1}$ :

$$
\hat{T}_{i}=T_{i-1}+B_{i} Q_{i-1}-\left(G_{i}+A_{i}\right) S_{i-1}
$$

Then for a preliminary estimate $\hat{S}_{10}$ of sources at depth $z_{i}$ :

$$
\begin{gathered}
\hat{S}_{i 0}=\rho C\left[v_{r i} \hat{Q} / K-\mathbf{v}_{H i} \cdot \nabla_{H} \hat{T}_{t}\right]+R \\
\hat{S}_{i 0}=\rho C\left[v_{r i}\left\{Q_{t-1}-\left(S_{i-1}+\hat{S}_{i 0}\right) \Delta z / 2\right\} K-\mathbf{v}_{H i} \cdot \nabla_{H} \hat{T}_{t}\right]+R
\end{gathered}
$$

where the subscript $H$ denotes horizontal components and $R$ represents the intrinsic sources, radioactivity plus cooling:

$$
R=q_{R}-\rho C \partial T / \partial t
$$

Solving (16) for $\hat{S}_{10}$ :

$\hat{S}_{0}=\frac{\left[\rho C\left\{v_{r l}\left(Q_{t^{-1}}-S_{i-1} \Delta z / 2\right) / K-\mathbf{v}_{H_{1}} \cdot \nabla_{H} \hat{T}_{l}\right\}+R\right]}{\left[1+\rho C v_{r t} \Delta z / 2 K\right]}$

In (16), (18), and hereafter the depth subscript $i$, etc., is dropped on a priori quantities such as $\rho, C, K$, and $R$ when they pertain to the same depth $z_{1}$ as the quantity on the left of the equation.

The denominator in (18) thus appears to set a limit for this procedure of

$$
\Delta z<-2 K / \rho C v_{r}
$$

which, for example, is $\sim 0.6 \mathrm{~km}$ in a subduction zone of $v_{r}=$ $-100 \mathrm{~mm} / \mathrm{yr}$.

However, regions which approach violation of (19) are constrained by other requirements detailed below.

The estimates $\hat{S}_{10}$ from (18) and $\hat{Q}_{i}$ from (11)

$$
\hat{Q}_{i}=Q_{1-1}-\left(S_{1-1}+\hat{S}_{i 0}\right) \Delta z / 2
$$

will be incomplete for a given size $\Delta s$ of area means (or corresponding maximum wave number $l_{x}$ ) because velocities $v$ and temperature gradients $\nabla_{H} \hat{T}_{1}$ and $\hat{Q}_{t} / K$ are calculated from mean values of areas of dimension $\Delta s$. There will necessarily be significant contributions from shorter-wavelength components to the vertical heat transport $(Q+$

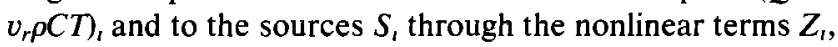
(4). These contributions require further constraints to determine $S$, and thence $Q_{1}$ and $T_{i}$. Plausible constraints are numbered 1 through 5 in the following paragraphs.

1. The energy flow in and out of the box of thickness $\Delta z$, north-south length $\Delta s$, east-west length $\sim \sin \theta \Delta s$, must balance. But lateral energy flows above the truncation level make it impossible to impose this condition locally; it is rigorous only for the entire globe:

$\int_{S}\left(\rho C v_{r 1} T_{1}+H_{1}+Q_{1}\right) r_{l}^{2} d S=\int_{S}\left[\left(\rho C v_{r i-1} T_{1-1}\right.\right.$

$\left.\left.+H_{i-1}+Q_{i-1}\right)\left(r_{t}+\Delta z\right)^{2}-\int_{z_{t-1}}^{z_{l}} R r^{2} d z\right] d S=Q_{G}$

where the integration is over the unit sphere

$$
H_{i}=\rho C\left[\left(v_{r}\right)_{i}-v_{r i} T_{i}\right]
$$

the advected heat transfer from above the truncation level, and $Q_{G}$ is the total heat transfer upward through level $i$.

2. However, since $\Delta z \ll \Delta s$, the contributions to the local energy balance from above the truncation level at depth $z_{l}$ should not differ greatly from that obtained by imposing the integrands of (21) locally, i.e.,

$$
\begin{aligned}
\hat{H}_{i}=\left(\frac{r_{i}+\Delta z}{r_{i}}\right)^{2} & {\left[\rho C v_{r-1} T_{i-1}+H_{i-1}+Q_{t-1}\right] } \\
& +\frac{1}{r_{i}^{2}} \int_{z_{t-1}}^{z_{t}} R r^{2} d z-\rho C v_{r i} \hat{T}_{i}-\hat{Q}_{1}
\end{aligned}
$$

3. If the source term $S_{t-1}$ at level $i-1$ differed from $\hat{S}_{(1-1) 0}$ as calculated by (18), then the estimated difference at level $i$ should be the same, i.e.,

$$
\hat{S}_{i}=\hat{S}_{t 0}+S_{t-1}-\hat{S}_{(t-1) 0}
$$


4. If there are no temperature reversals, then by (12)(13) the heat flow $Q$, should not be less than some minimum $Q_{m} \geq 0$. Adherence to such a minimum gradient at greater depths implies $S_{t+1}=0$ by (12), whence, assuming $\Delta z$ and $K$ constant,

$$
S_{t}=\left(Q_{1-1}-Q_{m}\right) / \Delta z-S_{l_{-1}} / 2
$$

However, sometimes this procedure resulted in numerical instability. Hence the source term $S$ was assumed to vary nonlinearly between $z_{t-1}$ and $z_{t}$, and $S_{i}$ was set at 0 when $Q$ was set at $Q_{m}$.

The most evident choice for the minimum flow $Q_{m}$ is that corresponding to an adiabat. But in some locations this led to temperatures at the fully convecting level much too low to satisfy (1). Hence

$$
Q_{m}=K\left(T_{f}-T_{i}\right) /\left(z_{f}-z_{l}\right)
$$

was used, where $z_{f}$ is the fully convecting level and $T_{f}$ is the temperature there, obtained as described below in (29)-(31).

5 . The temperature $T$, should not be greater than some maximum $T_{x}$. Such a fixing of temperatures constrains not only the sources $S$ but the flow $Q$ as well, hence requiring a two-step extrapolation if the linear variation of $S$ across $\Delta z$ is imposed. The resulting awkward expression, from (12)-(13) (assuming $\Delta z$ and $K$ constant again), is

$$
S_{i}=\frac{T_{l-1}-3 B_{i} Q_{l-1}-\left(G_{i}+B_{i} \Delta z\right) S_{l-1}+\left(A_{t}+B_{i} \Delta z / 2+G_{i}\right)\left(Q_{a}+S_{t-1} \Delta z / 2-Q_{i-1}\right) / \Delta z-T_{x}}{B_{i} \Delta z / 2-A_{i}}
$$

where $Q_{a}$ is the flow $K \alpha g T / C$ along the adiabat. Again, numerical instabilities resulted, so $S$ had to be assumed to vary nonlinearly over $\Delta z$ to $S=0$ where $Q=Q_{a}$ and $T=T_{\mathrm{r}}$ :

$$
T_{x}=T_{f}-Q_{a}\left(z_{f}-z_{i}\right) / K
$$

The temperatures $T_{f}$ at the fully convecting level satisfying (1) were obtained by a minimum normed sum procedure, using a Lagrangian multiplier:

$$
\begin{aligned}
F=\int_{S}\left|T-T_{0}\right|^{n} r^{2} d S & +n \lambda\left\{Q_{G}-\rho C\right. \\
& \left.\cdot \int_{S} v_{r}\left(T-T_{0}\right) r^{2} d S\right\}=\min
\end{aligned}
$$

where $T_{0}$ is a global mean which must be prescribed, and integration is again over the unit sphere. Thence

$$
\lambda=Q_{G} /\left\{\rho C \int_{S}\left|v_{r}\right|^{n} r^{2} d S\right\}
$$

and

$$
T=T_{0}+\lambda \operatorname{sign}\left(v_{r}\right)\left|v_{r}\right|^{\prime \prime-1}=T_{f}
$$

Hence plausibility requires $n>1$. In a real convecting system with temperature-dependent viscosity dominated by subduction, possibly $n>2$ is appropriate.

Given that some of the source terms $S_{1}$ are constrained by the limits 4,5 , then the balance of the sources $S_{1}$ plus all the $H_{i}$, the advected heat transfers from above the truncation level, must be adjusted to satisfy condition 1, the global heat transfer equation (21). Again a minimum normed sum and Lagrangian multiplier procedure are indicated. Both $S_{\text {, and }}$ $H_{i}$ should be included in this normed sum, since both can plausibly differ from the estimates given by (23)-(24). What norm should be minimized is not at all clear, however. To simplify matters somewhat, it is assumed that the norm is quadratic, $n=2$, with a correlation coefficient $\nu$ between $S-S_{R}$ and $H-H_{R}$, where $S_{R}$ and $H_{R}$ are reference values. With these assumptions, the sum to be minimized becomes:

$$
\begin{aligned}
F= & \int_{A}\left\{\left[\frac{S-S_{R}}{\sigma_{S}}\right]^{2}-2 \nu \cdot \frac{\left(S-S_{R}\right)\left(H-H_{R}\right)}{\sigma_{S} \sigma_{H}}\right. \\
& \left.+\left[\frac{Q-Q_{R}}{\sigma_{Q}}\right]^{2}+\left[\frac{\left(T-T_{R}\right)}{\sigma_{T}}\right]^{2}\right\}^{2} d S \\
& +\int_{S}\left[\frac{H-H_{R}}{\sigma_{H}}\right]^{2} r^{2} d S \\
& +2 \lambda\left\{Q_{G}-\int_{S}\left[v_{r} \rho C\left(T-T_{0}\right)+H+Q\right] r^{2} d S\right\}=\min
\end{aligned}
$$

where $A$ denotes integration over the adjustable area only. A variety of reference values, $S_{R}, H_{R}, Q_{R}$, and $T_{R}$, could be argued: zeros, global means, or estimates by (18), (24), (12), (13), and (23). In addition it could be argued that for $T$ there is some transition between a "conductive" temperature

based on surface heat flow $Q$ and assumed intrinsic sources $R$ and a "convective" temperature based on that calculated for the fully convective level, (31). To give a smooth variation from $T_{0}, Q_{0}$ to $T_{f}$

$$
T_{R}=\left[\left\{R^{2}-\left(1-z / z_{f}\right)^{2}\right\}-\delta\right]\left[T_{f}-T_{0}\right]+T_{0}
$$

was actually employed, where

$$
\delta=\left(T_{f}-T_{0}\right) K / Q_{0} z_{f}
$$

and

$$
R=\left(1+\delta^{2}\right)^{1 / 2}+\left(1+\delta-\left(1+\delta^{2}\right)^{1 / 2}\right)\left(z / z_{f}\right)^{p}
$$

Also, the expected magnitudes $\sigma_{S}, \sigma_{H}, \sigma_{T}$, and $\sigma_{Q}$ of the variations are somewhat arbitrary but necessary, as in any procedure where a normed sum is minimized. Using (12)(13) to eliminate $Q$ and $T$ and $\partial F / \partial H=0$ to eliminate $H$, the requirement $\partial F / \partial S=0$ for any point within the adjustable area leads to the form

$$
S(\theta, \varphi)=[P(\theta, \varphi)-\mathrm{E}(\theta, \varphi) \lambda] / h
$$

where

$$
\begin{gathered}
h=\left(1-\nu^{2}\right) / \sigma_{S}^{2}+D_{i}^{2} / \sigma_{Q}{ }^{2}+A_{t}^{2} / \sigma_{T}^{2} \\
P=\frac{1-\nu^{2}}{\sigma^{2}(S)} S_{R}+\frac{D_{t}}{\sigma_{Q}^{2}}\left(Q_{t-1}-S_{t-1} \Delta z / 2-Q_{R}\right) \\
+\frac{A_{t}}{\sigma_{T}^{2}}\left(T_{i-1}-B_{l} Q_{i-1}-G_{i} S_{t-1}-T_{R}\right) \\
E=v_{r} \rho C A_{t}+\frac{\Delta z}{2}-\nu \sigma_{H} / \sigma_{S}
\end{gathered}
$$




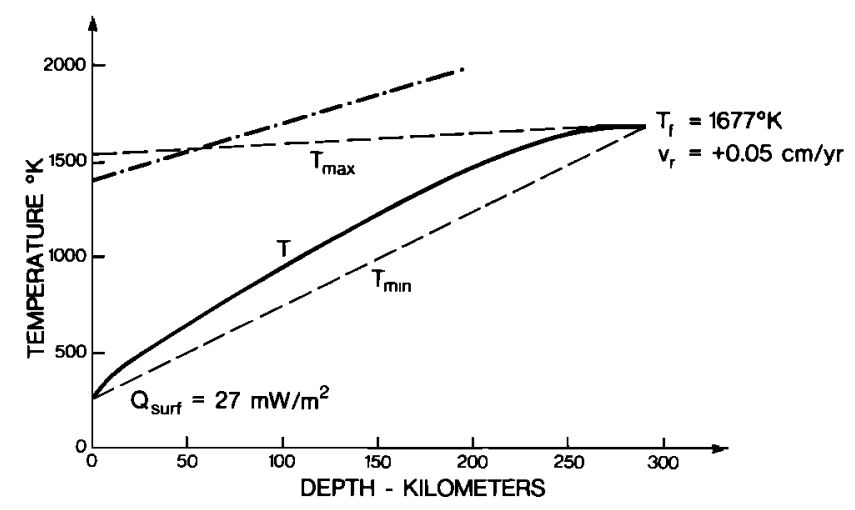

Fig. $1 a$

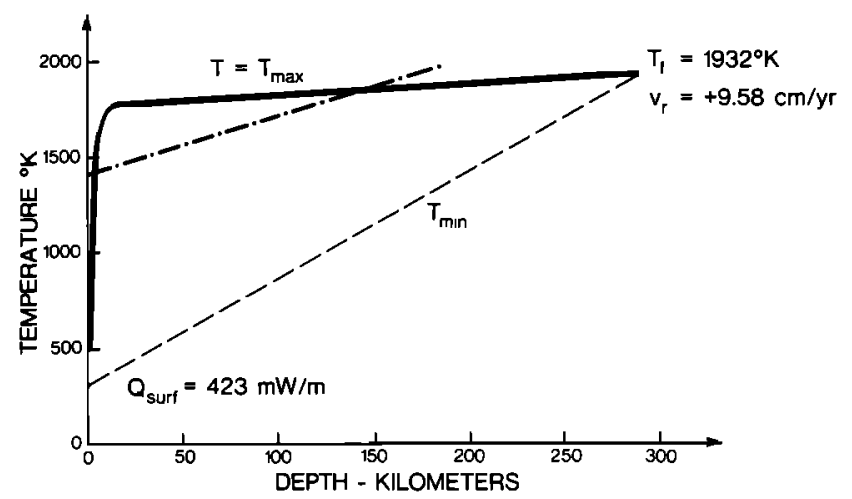

Fig. 1c

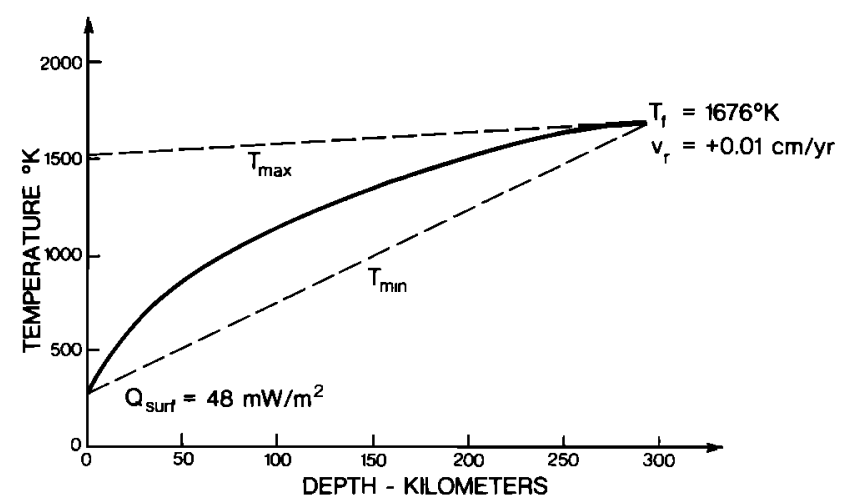

Fig. $1 b$

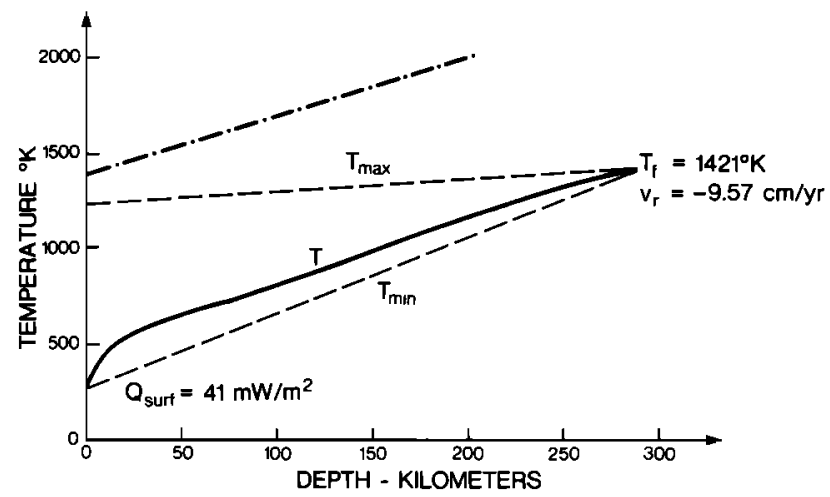

Fig. 1d

Fig. 1. Examples of temperature profiles: (a) ocean basin; South Atlantic, $42.5^{\circ} \mathrm{S}, 22.5^{\circ} \mathrm{E} ;(b)$ stable continent: Africa, $17.5^{\circ} \mathrm{S}, 32.5^{\circ} \mathrm{E} ;(c)$ ocean rise; Southeast Pacific, $22.5^{\circ} \mathrm{S}, 112.5^{\circ} \mathrm{W}$ (Note that the temperature curve should be even further reduced at shallow depth if it is to remain below the basalt solidus, the dot-dash line.); (d) subduction zone; Japan, $37.5^{\circ} \mathrm{N}, 142.5^{\circ} \mathrm{E}$.

in which the coefficients are defined by (14). The global condition (21) can be written

$$
\begin{aligned}
\int_{S}\left\{\rho C v_{r}\left(\hat{T}_{i}-T_{0}\right)\right. & \left.+\hat{H}_{i}+\delta H_{1}+\hat{Q}_{l}\right\} r_{i}^{2} d S \\
& +\int_{A}\left\{\rho C v_{r} \delta T_{i}+\delta Q_{i}\right\} r_{i}^{2} d S=Q_{G}
\end{aligned}
$$

where (using $\partial F / \partial H=0$ to obtain $H_{i}$ ):

$$
\begin{aligned}
& \delta T_{i}=T_{i}-\hat{T}_{i}=-A_{i} \delta S_{i} \\
& \delta q_{t}=Q_{t}-\hat{Q}_{i}=-\frac{\Delta z}{2} \delta S_{i} \\
& \delta H_{t}=H_{i}-\hat{H}_{i}=H_{R}-\hat{H}_{i}+\nu \frac{\sigma_{H}}{\sigma_{R}}\left(\hat{S}_{i}+\delta S_{i}-S_{R}\right)+\sigma_{H}{ }^{2} \lambda
\end{aligned}
$$$$
\text { inside } A
$$

$$
\begin{aligned}
& \delta H_{t}=H_{i}-\hat{H}_{t}=H_{R}-\hat{H}_{i}+\sigma_{H}{ }^{2} \lambda \quad \text { outside } A \\
& \delta S_{t}=S_{i}-\hat{S}_{i}=(P-E \lambda)-\hat{S}_{i}
\end{aligned}
$$

Using (39) to eliminate everything in favor of the estimates $\hat{T}_{l}, \hat{Q}_{l}, \hat{S}_{l}$, and $\lambda$ in (38) obtains

$\lambda=\left[Q_{G}-\int_{S}\left\{\rho C v_{r}\left(\hat{T}_{i}-T_{0}\right)+H_{R}+\hat{Q}_{\}}\right\} r^{2} d S\right.$

$$
\begin{aligned}
& +\int_{A}\left\{\left(\rho C v_{r} A_{i}+\frac{\Delta z}{2}\right)\left(P-\hat{S}_{i}\right)\right. \\
& \left.\left.-\nu \frac{\sigma_{H}}{\sigma_{R}}\left(P-S_{R}\right)\right\} r^{2} d S\right] /\left[\int_{S} \sigma_{H}{ }^{2} r^{2} d S\right. \\
& \left.+\int_{A}\left(E^{2} / h\right) r^{2} d S\right]
\end{aligned}
$$

Then the sources $S_{1}$ are calculated from (36), the temperature $T$, and heat flow $Q_{1}$ by (12)-(13), and the supertruncation contribution $H$, by (39).

In summary the temperature was determined by the energy equation, subject to the global condition, so long as it stayed within the limits defined by (26) and (28). Four examples are shown in Figure 1. A convenient parameter for characterizing a location is $\delta$ defined by (34): the ratio of temperature rise to the fully convecting depth $z_{f}$ to the linear extrapolation from the surface heat flow $Q_{0} z_{f} / K$. This ratio varied from about 0.05 at rises to 1.05 in regions of low measured $Q_{0}$.

\section{DATA}

The plate velocities used were those of Minister and Jordan [1978]; the plate margins, those of Kaula [1975b]. The heat flow data were the $5^{\circ}$ means of Chapman and Pollack [1980]: observed values extrapolated by correlation with tectonic setting age, as described in Chapman and Pollack [1975]. A significant change in this compilation 


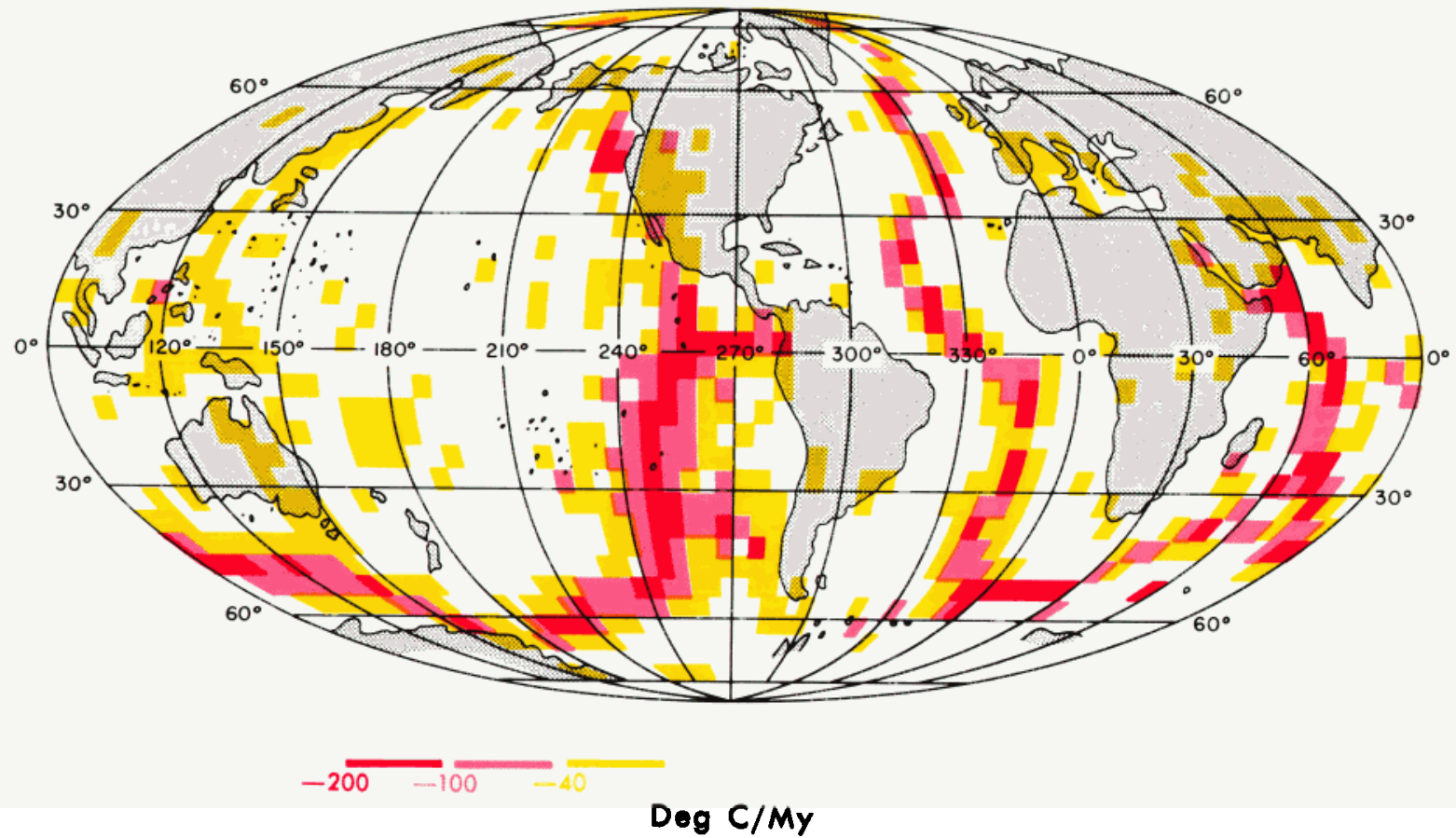

Plate 1. Effective sources $-S / \rho C$, in ${ }^{\circ} \mathrm{C} / \mathrm{m}$.y. averaged over the uppermost $10 \mathrm{~km}$. As discussed in the text, the large values arise from advection, $\mathbf{v} \cdot \nabla T$

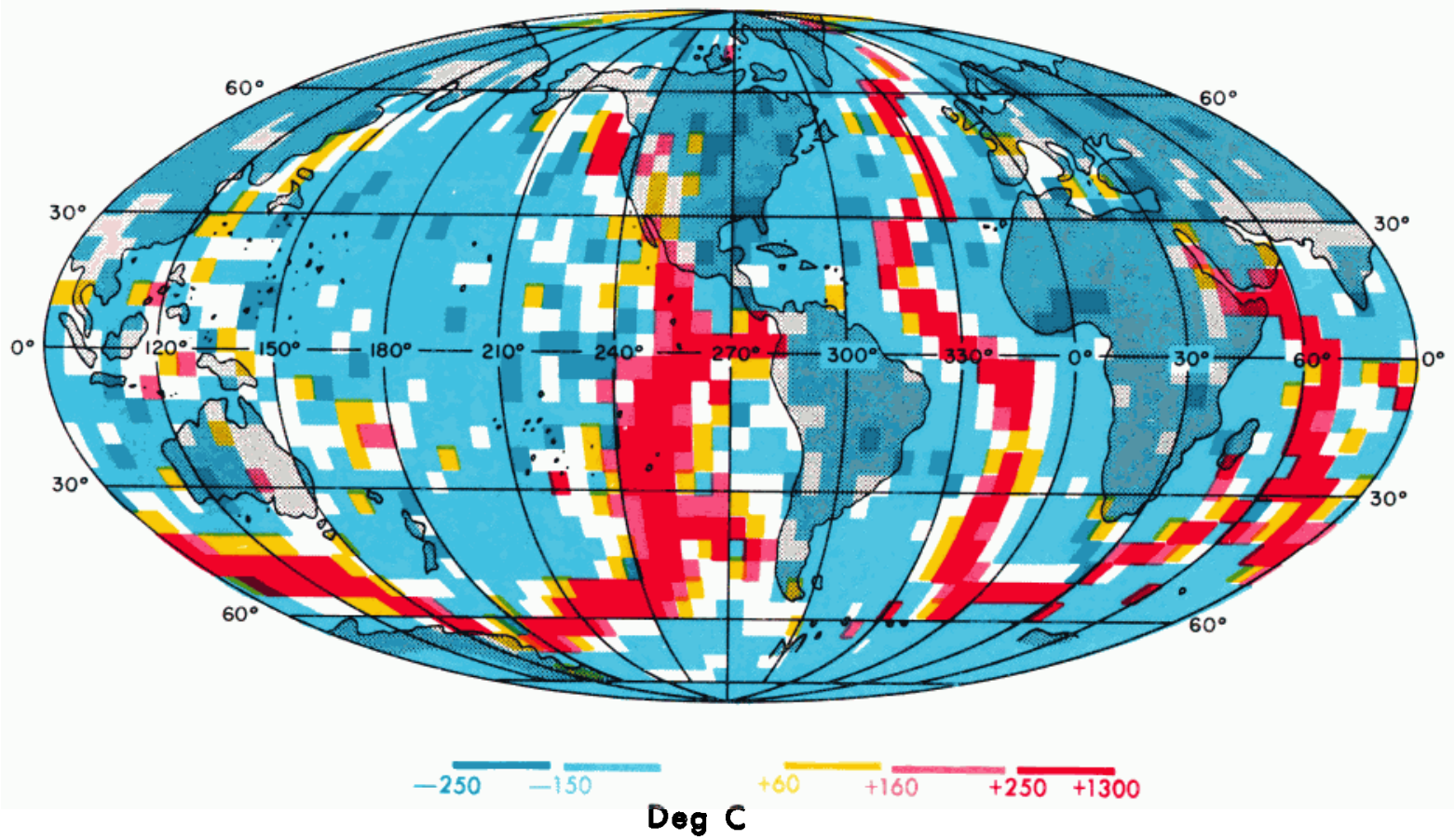

Plate 2. Temperatures at 10-km depth; they are dependent almost entirely on the prescribed surface heat flow less source effects in the hottest regions. 


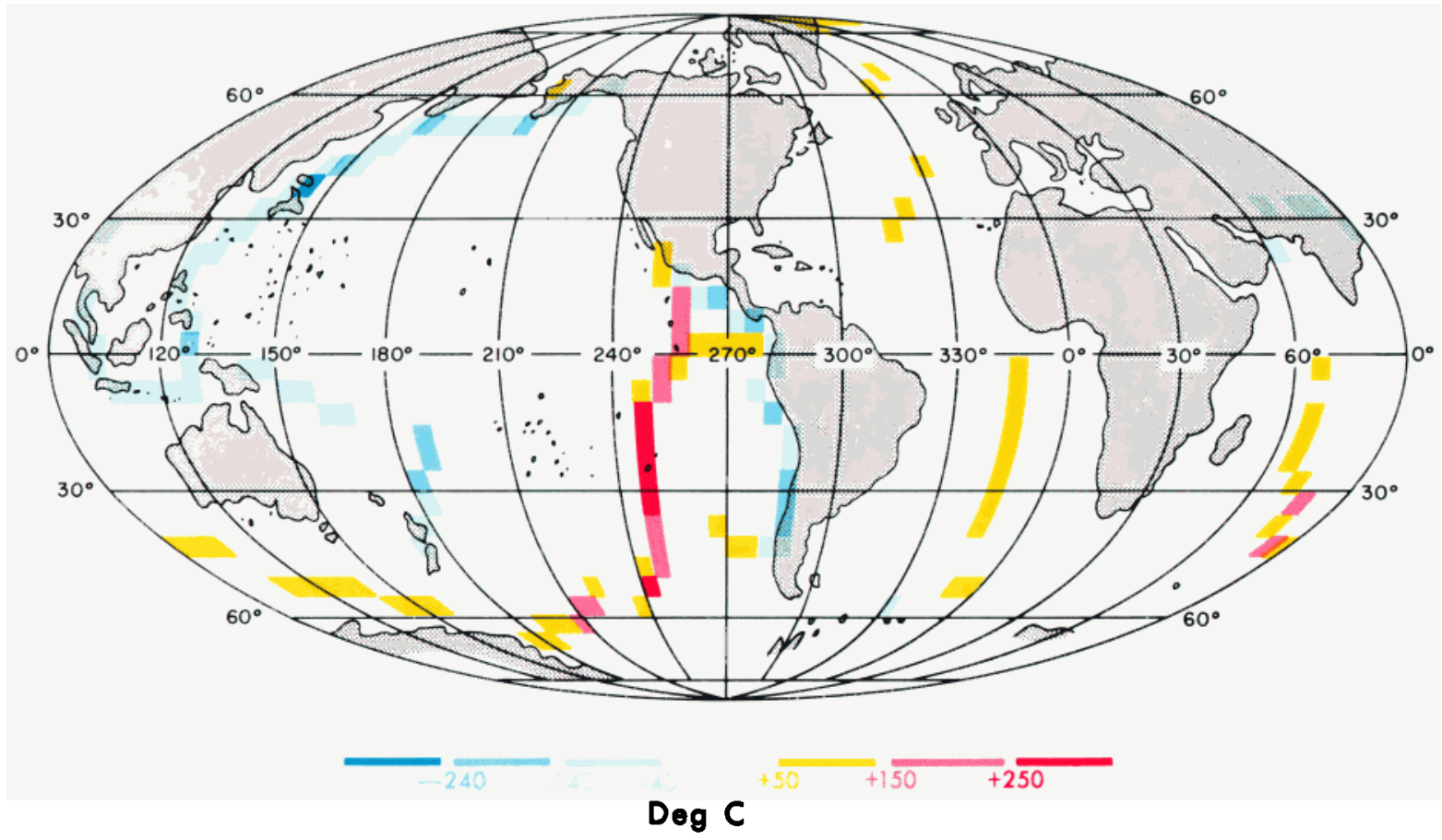

Plate 3. Temperatures at $280-\mathrm{km}$ depth, which are dependent on the velocity field integrated down from the surface; a mean temperature of $1676^{\circ} \mathrm{K}$; a mean heat transfer of $67 \mathrm{~mW} / \mathrm{m}^{2}$; expression of velocities and temperatures as $5^{\circ}$ square means; and the optimization of (29) with the norm $n=2.0$.

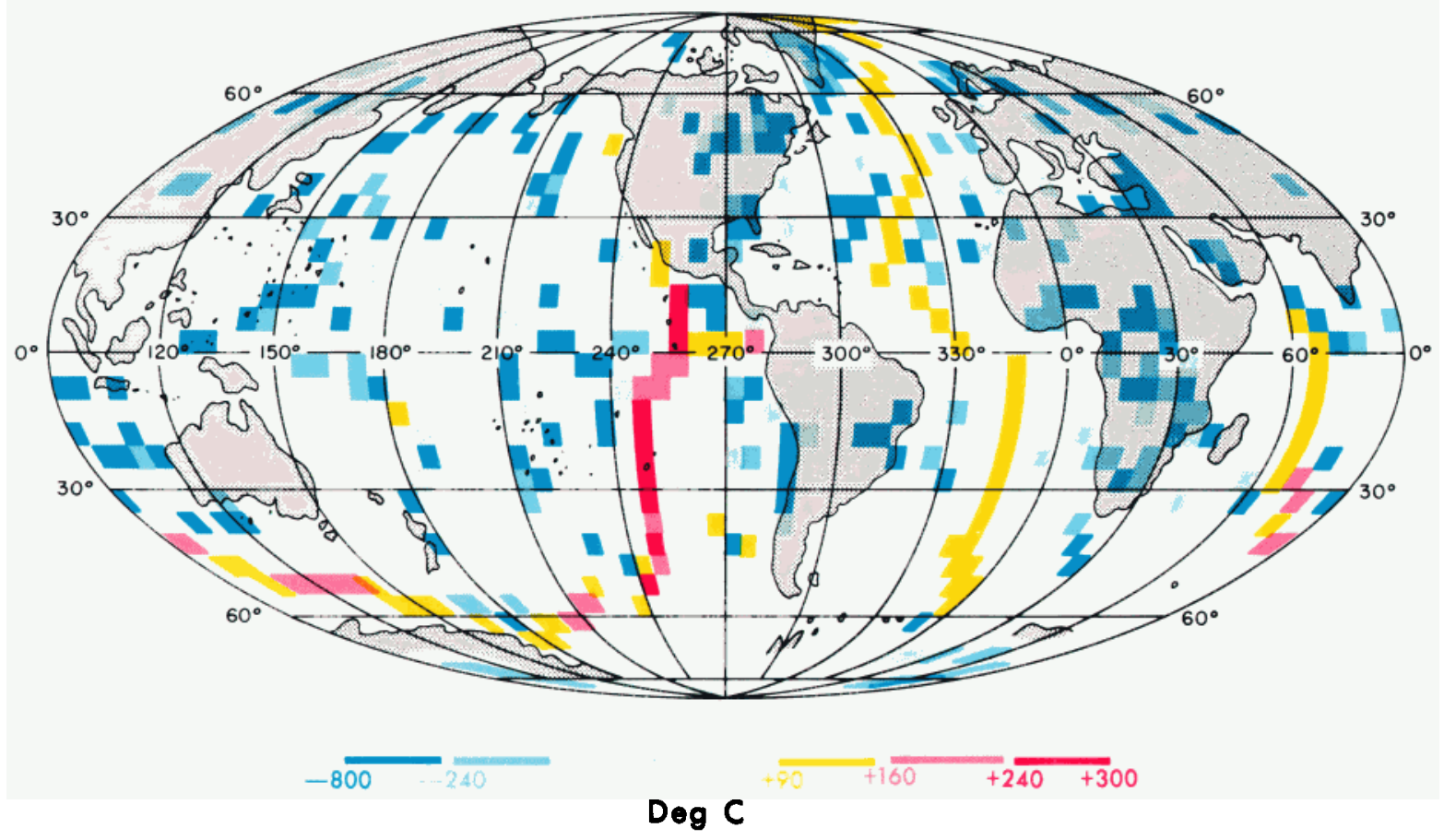

Plate 4. Temperatures at $100-\mathrm{km}$ depth, which are dependent on integration of the energy equation down from the surface plus limitations based on the temperatures at the fully convecting depth of $280 \mathrm{~km}$. 
TABLE 1. Solution Parameter Values

\begin{tabular}{lcccc}
\hline \multicolumn{1}{c}{ Parameter } & Symbol & $\begin{array}{c}\text { Defining } \\
\text { Equation }\end{array}$ & Unit & Value(s) \\
\hline $\begin{array}{l}\text { Norm for fully convecting } \\
\text { level }\end{array}$ & $n$ & $(29)$ & - & $1.5,2.0,2.5$ \\
$\begin{array}{l}\text { Standard Derivations } \\
\quad \text { Sources }\end{array}$ & & & & \\
$\quad$ Heat Transfer & $\sigma_{S}$ & $(32)$ & $\mathrm{W} / \mathrm{m}^{3}$ & $1.0 \times 10^{-7}$ \\
$\quad$ Subtruncation & $\sigma_{Q}$ & $(32)$ & $\mathrm{W} / \mathrm{m}^{2}$ & $3.0 \times 10^{-3}$ \\
$\quad$ Supertruncation & $\sigma_{H}$ & $(32)$ & $\mathrm{W} / \mathrm{m}^{2}$ & $3.0 \times 10^{-3}$ \\
$\quad \begin{array}{l}\text { Temperature } \\
\text { Correlation }\end{array}$ & $\sigma_{T}$ & $(32)$ & ${ }^{\circ} \mathrm{C}$ & 30.0 \\
$\quad$ Sources and heat transfer & $\nu$ & $(32)$ & - & 0.5 \\
$\begin{array}{l}\text { Temperature curve expo- } \\
\text { nent }\end{array}$ & $p$ & $(35)$ & - & 1.1 \\
\hline
\end{tabular}

between 1975 and 1980 was to increase the estimate in regions of rapid spreading to allow for hydrothermal circulation at rises. The topography entered into our calculation only to define the thickness of the continental crust. The margin between continent and ocean was taken as the minimum in the hyposometric curve, about $1.5 \mathrm{~km}$ below sea level.

The continental crustal thickness was calculated by assuming Airy isostasy. The continental heat source content $q_{R}$ followed the model of Chapman and Pollack [1975] and Pollack and Chapman [1977]. The oceanic crustal thickness was assumed to be $6 \mathrm{~km}$ and the heat source content $q_{R}$ to be $10^{-7} \mathrm{~W} / \mathrm{m}^{3}\left(10^{-6} \mathrm{ergs} / \mathrm{cm}^{3} / \mathrm{s}\right)$.

The mean temperatures and material parameters (density, thermal expansivity, thermal diffusivity, and viscosity) used were those given in Table 4 of Kaula [1980]. In addition, an intrinsic heat source density $\left(q_{R}-\rho C \partial T / \partial t\right)$ of $2.5 \times 10^{-8}$ $\mathrm{W} / \mathrm{m}^{3}$ in the mantle was assumed. From a purely spatial integration, such as in this paper, there is no way to distinguish radioactive sources from cooling. Such a discrimination requires a temporal integration as well.

\section{Computations and Results}

Aside from the surface field values and material properties outlined in the previous section, there are only seven neces- sary parameters for the model developed here. These are given in Table 1. However, of these parameters, only the fully convecting level norm exponent $n$ was found to have a significant effect, since the others act only through the levelby-level adjustment equation (32). For the reference values $S_{R}, H_{R}$, and $Q_{R}$ the extrapolated estimates (24), (23), and (20) were used. For $T_{R}$ the smooth curve (33) was used.

Table 2 summarizes the effects of varying the fully convecting level norm $n$. The greater value of $n=2.5$ leads to implausibly large temperature extremes around rises, while the lesser value of 1.5 leads to an implausibly choppy temperature pattern, in part an exaggeration of "Gibbs effects" in the velocity field. All solutions obtain a maximum temperature range of $\sim 1500^{\circ} \mathrm{C}$ at about $10-\mathrm{km}$ depth, a rather unavoidable consequence of the extraordinary range from 16 to $423 \mathrm{~mW} / \mathrm{m}^{2}$ in observed heat flow. This temperature range is greater than is petrologically permissible, as indicated in Figure 1c. Given the constraint of an adiabat to the fully convecting level, there necessarily must be an extremum of source terms $S$ or advection-approximately $-S / \rho C$ by (5) and (10)_at very shallow depth below regions of high heat flow. Most notable is the southeast Pacific Rise, where the advection must average about $-200 \% \mathrm{~m}$.y. in the top $10 \mathrm{~km}$. (In contrast, typical oceanic crust radioactivity, $-q_{R} / \rho C$ is only $-10 \% \mathrm{~m}$.y. and the mean intrinsic sources of the mantle,

TABLE 2. Summary of Solution Propertıes

\begin{tabular}{|c|c|c|c|c|c|c|}
\hline \multirow[b]{2}{*}{ Property } & \multicolumn{6}{|c|}{ Depth, km } \\
\hline & 10 & 20 & 50 & 100 & 180 & 280 \\
\hline \multicolumn{7}{|c|}{ Temperature, ${ }^{\circ} \mathrm{K}$} \\
\hline 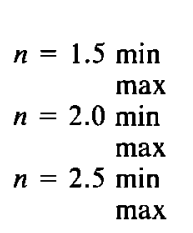 & $\begin{array}{r}-213 \\
+1208 \\
-234 \\
+1312 \\
-234 \\
+1319\end{array}$ & $\begin{array}{c}\text { Extreme } \\
-387 \\
+965 \\
-387 \\
+1048 \\
-467 \\
+1137\end{array}$ & $\begin{array}{l}\text { ative to } \\
-699 \\
+532 \\
-700 \\
+616 \\
-701 \\
+706\end{array}$ & $\begin{array}{l}\text { I } \\
-802 \\
+233 \\
-809 \\
+371 \\
-809 \\
+407\end{array}$ & $\begin{array}{l}-528 \\
+193 \\
-545 \\
+280 \\
-592 \\
+373\end{array}$ & $\begin{array}{l}-178 \\
+185 \\
-255 \\
+274 \\
-322 \\
+369\end{array}$ \\
\hline $\begin{array}{c}n=2.0 \\
\text { mean } \\
\max \end{array}$ & $\begin{array}{r}1.3 \\
66.6 \\
376.3\end{array}$ & $\begin{array}{r}\text { Condu } \\
1.1 \\
52.1 \\
224.7\end{array}$ & $\begin{array}{r}\text { low, } m V \\
1.2 \\
36.3 \\
109.0\end{array}$ & $\begin{array}{r}1.5 \\
8.5 \\
62.9\end{array}$ & $\begin{array}{r}1.9 \\
3.0 \\
33.4\end{array}$ & $\begin{array}{l}2.0 \\
2.4 \\
2.7\end{array}$ \\
\hline \multicolumn{7}{|c|}{ Convected Flow, $\mathrm{mW} / \mathrm{m}^{2}$} \\
\hline $\begin{array}{c}n=2.0 \text { min } \\
\text { mean } \\
\text { max }\end{array}$ & $\begin{array}{r}-55.5 \\
2.1 \\
+88.5\end{array}$ & $\begin{array}{r}\text { Sou } \\
-301.3 \\
-1.4 \\
+36.6\end{array}$ & $\begin{array}{r}10^{-7} \mathrm{~W} / \mathrm{m} \\
-38.1 \\
-0.3 \\
+13.5\end{array}$ & $\begin{array}{l}-6.0 \\
-0.0 \\
+6.2\end{array}$ & $\begin{array}{r}-1.3 \\
0.0 \\
+1.0\end{array}$ & $\begin{array}{l}0.0 \\
0.0 \\
0.0\end{array}$ \\
\hline
\end{tabular}


$-q_{R} / \rho C+\partial T / \partial t$, are about $-0.2 \%$ m.y.) In the formalism of this paper the large local convective heat transfer associated with partial melting, etc., must be nonlinear variation in sources $S$ between level $z_{i-1}$ and level $z_{l}$ at which the maximum condition (28) is satisfied. From (12):

$$
\langle S\rangle_{t-1 / 2}=\left(Q_{t-1}-Q_{i}\right) / \Delta z
$$

Plate 1 is a plot of these averaged sources converted to units of advection $\left({ }^{\circ} \mathrm{C} / \mathrm{m}\right.$.y.) by application of the factor $-1 / \rho C$. The maximum averaged advection decreases from $190 \% \mathrm{~m} . \mathrm{y}$. in the top $10 \mathrm{~km}$ to $130 \% \mathrm{~m} . \mathrm{y}$. over $10-20 \mathrm{~km}$ depth, $50 \% \mathrm{~m}$.y. over $40-50 \mathrm{~km}, 16 \% \mathrm{~m} . \mathrm{y}$. over $90-100 \mathrm{~km}, 6 \% \mathrm{~m} . \mathrm{y}$. over 180-200 km depth, etc.

Plates 2-4 give the temperatures at depths of $10 \mathrm{~km}, 280$ $\mathrm{km}$, and $100 \mathrm{~km}$. The most implausible features in these plots, the very low temperatures $\sim 1000 \mathrm{~km}$, of $\sim 15 \mathrm{~m}$.y., off rapidly spreading rises, are the consequence of low observed heat flows: for example, $32 \mathrm{~mW} / \mathrm{m}^{2}$ at $-2.5^{\circ}$ latitude, $237.5^{\circ}$ longitude; $34 \mathrm{~mW} / \mathrm{m}^{2}$ at $-325^{\circ}, 92.5^{\circ}$. These are probably the result of measurements in regions of permeable sediments where the temperature gradient is depressed by hydrothermal circulation [Anderson et al., 1977; Sclater et al., 1980; Anderson and Skilbeck, 1981].

As discussed in the introduction, judgment as to the extent that these irregular appearing patches of low temperature are real must depend on more detailed examination of heat flow observations.

\section{ConClusions}

The fundamental premises of this paper are:

1. The velocity field to depth $280 \mathrm{~km}$ does not differ significantly from an extrapolation from the surface field that takes into account only radial variation in viscosity and density.

2. Eighty-six percent of the heat flow out of the earth, 4 $\times 10^{13} \mathrm{~W}$, comes from below $30 \mathrm{~km}$.

3. The heat transfer at $280-\mathrm{km}$ depth is entirely convective.

4. The temperature gradient with respect to depth is everywhere at least adiabatic: $\partial T / \partial z \geq \alpha g T / C$.

5. The surface heat flow field of Chapman and Pollack [1980] is correct.

6. The surface velocity field of Minster and Jordan [1978] is correct.

7. The material parameters and mean temperatures of Kaula [1980] are correct.

8. The temperature and velocity fields are adequately represented by $5^{\circ}$ square means.

Given these premises, the following inferences seem rather ineluctable:

1. The greatest lateral differences in temperature, $\sim 1500^{\circ} \mathrm{C}$, occur within the top $20 \mathrm{~km}$.

2. The greatest nonlinear terms in the energy equation, $\sim 200^{\circ} \mathrm{C} / \mathrm{m}$.y., occur within the top $20 \mathrm{~km}$.

3. Below $\sim 50-\mathrm{km}$ depth, the greatest departures in temperature from the mean are negative "tongues," which reach an extreme of about $-825^{\circ} \mathrm{C}$ at depth $\sim 100 \mathrm{~km}$.

4. Below $\sim 50-\mathrm{km}$ depth, heat transfer is more convective than conductive.

5. At the fully convecting level of $\sim 280 \mathrm{~km}$, temperature variations are at least $\pm 180^{\circ} \mathrm{C}$ about the mean.

These inferences are not sensitive to the one major parameter left free by the premises, the norm $n$ on the fully convecting level condition, (29). The nonlinear terms more that $10 \% \mathrm{~m} . \mathrm{y}$. over the oceans must be advection, $\mathrm{v} \cdot \nabla T$, rather than dissipation, $\eta \dot{e}^{2} / 2 \rho C$, which for plausible strain rates averaged over $\gtrsim 100 \mathrm{~km}$ and $3 \times 10^{-7}$ yr [Kaula, 1980] could hardly be more than $10 \% \mathrm{~m}$.y. See McKenzie and Jarvis [1980] for a similar conclusion based on thermodynamic arguments.

Thus it remains to question the premises.

Premise 1 implies negligible effect of shallow inhomogeneities on the velocity and may seem at first sight to contradict various force balance models involving "slab pulls," "ridge push," etc. [e.g., Forsyth and Uyeda, 1975]. However, these models are incorporated by starting from the observed surface velocities; the premise is more accurately stated as having no significant nonlinear departures from the flows inferred from the surface velocities.

Premise 2 regarding heat sources is really that $50 \%$ of the heat outside that convectively transported comes from below $30 \mathrm{~km}$, since about $70 \%$ of the total is lost by cooling of the spreading oceanic lithosphere [Sclater et al., 1980].

Premise 3, a fully convecting level, is contrary to indications of the tectosphere of Jordan [1979] if applied at a depth of $280 \mathrm{~km}$, since at this depth there may still be perceptible conductive transfer under old continents. However, these regions are of slight contribution to the global heat budget. More serious, the implementation of the fully convecting level hypothesis at depth $280 \mathrm{~km}$ by the optimizing condition (29), with uniform norm $n$, lead to temperatures which may be too high in subduction zones (but it should be recalled that these temperatures are $5^{\circ}$ block means). Application at a greater depth, however, would make premise 1 (no effect on the velocity field of lateral inhomogeneities, etc.) more wrong. A compromise might be to use a higher norm $n$ for temperatures below the mean, $T<T_{0}$, than for those above.

Premise 4 regarding the limit on temperature gradients is somewhat arbitrary; it does not come from physical necessity. But, as indicated by boundary layer theory [Olson and Corcos, 1980], contradictions are of limited extent and are not inferable from more detailed examination of observations [Lewis, 1981]. In any case, their global significance is slight.

Premise 5, the correctness of the surface heat flow field, is the most questionable because of the data sparsity. Ironically, the most dubious low temperatures probably arise from areas of some data, but limited and nonrepresentative (because of hydrothermal circulation, etc.), rather than areas of no data at all, where the plausible extrapolation based on age and tectonic setting was used by Chapman and Pollack [1975, 1980].

Premise 6, (the velocity field) is as sure as the plate tectonic hypothesis, while Premise 7 (material parameters) is essentially a corollary of premise 1: given the kinematics, it takes an appreciable depth-more than $280 \mathrm{~km}$-for physical effects to make a perceptible difference.

Premise 8 (representation by $5^{\circ}$ square means) appears to be quite questionable. A harmonic analysis of the radial velocity $v_{r}$ led to a rather slowly decreasing spectrum. Hence an appreciable portion of the heat transfer $v, \Delta T$ could be provided by variations of shorter scale than $5^{\circ}$.

Inferences 1-4 stated above thus appear to be rather firm. But the temperature variations at the fully convecting level, $\pm 180^{\circ} \mathrm{C}$, may be too high.

All the inferences are adumbrated in earlier studies. The 
temperatures under stable areas, Figure $1(a, b)$, fall within the range of earlier studies, such as Sclater et al. [1980]. The high-temperature differences and advection in the top $20 \mathrm{~km}$ are necessary implications of petrological studies of ocean rise conditions, such as Bottinga and Allégre [1978], as well as more detailed geophysical analyses, such as Lewis [1981]. The temperatures about $800^{\circ} \mathrm{C}$ below the mean at $\sim 100-\mathrm{km}$ depth occur in thermal models of subduction zones, such as Toksöz et al. [1971] and Bird [1978]. However, a global solution, although unavoidably crude, is necessary to connect surface-data-based crust and lithosphere models to more comprehensive mantle convection studies.

Acknowledgments. This work was supported by NASA grant NSG-5263. H. N. Pollack, University of Michigan, kindly provided the heat flow data. Helpful comments were received from N. H. Sleep, Stanford University, and two anonymous referees.

\section{REFERENCES}

Anderson, R. N., and J. N. Skilbeck, Oceanic heat flow, in The Oceanic Lithosphere, The Sea VII, edited by C. Emiliani, pp. 489-523, John Wiley, New York, 1981.

Anderson, R. N., M. G. Langseth, and J. G. Sclater, The mechanism of heat transfer through the floor of the Indian Ocean, $J$. Geophys. Res., 82, 3391-3409, 1977.

Bird, P., Stress and temperature in subduction shear zones: Tonga and Mariana, Geophys. J. R. Astron. Soc., 55, 411-434, 1978.

Bottinga, Y., and C. J. Allégre, Partial melting under spreading ridges, Phil. Trans. R. Soc. Lond. A., 288, 501-525, 1978.

Chapman, D. S., and H. N. Pollack, Global heat flow: A new look, Earth Planet. Lett., 28, 23-32, 1975.

Chapman, D. S., and H. N. Pollack, Global heat flow: Degree 18 spherical harmonic representation, EOS Trans. AGU, 61, 383, 1980.

Embley, R. W., M. A. Hobart, R. N. Anderson, and D. Abbott, Anomalous heat flow in the northwest Atlantic: A case for continued hydrothermal circulation in 80-m.y. crust, J. Geophys. Res., 88, 1067-1074, 1983.

Forsyth, D., and S. Uyeda, On the relative importance of the driving forces of plate motion, Geophys. J. R. Astron. Soc., 43, 163-200, 1975.

Hager, B., and R. J. O'Connell, Subduction zone dip angles and flow driven by plate motion, Tectonophysics, 50, 111-138, 1978.

Hager, B., and R. J. O'Connell, Kinematic models of large-scale flow in the earth's mantle, J. Geophys. Res., 84, 1031-1048, 1979.
Hager, B., and R. J. O'Connell, A simple global model of plate dynamics and mantle convection, J. Geophys. Res., 86, 48434867,1981 .

Jordan, T. H., The deep structure of the continents, Sci. Am., 240(1), 92-107, 1979.

Kaula, W. M., Product-sum conversion of spherical harmonics with application to thermal convection, J. Geophys. Res., 80, 225-231, $1975 a$.

Kaula, W. M., Absolute plate motions by boundary velocity minimizations, J. Geophys. Res., 80, 244-248, 1975b.

Kaula, W. M., Extensions of the theory of statistical analysis on a sphere, Boll. Geod. Sci. Aff., 37, 495-505, 1978.

Kaula, W. M., Material properties for mantle convection consistent with observed surface fields, J. Geophys. Res., 85, 7031-7044, 1980.

Lewis, B. T. R., Isostasy, magma chambers, and plate driving forces on the East Pacific Rise, J. Geophys. Res., 86, 4868-4880, 1981.

McKenzie, D. P., and G. Jarvis, The conversion of heat into mechanical work by mantle convection, J. Geophys. Res., 85, 6093-6096, 1980.

McKenzie, D. P., M. J. Roberts, and N. O. Weiss, Convection in the earth's mantle: Toward a numerical simulation, J. Fluid Mech., 62, 465-538, 1974.

Minister, J. B., and T. H. Jordan, Present-day plate motions, $J$. Geophys. Res., 83, 5331-5334, 1978.

Olson, P., and G. M. Corcos, A boundary layer model for mantle convection with surface plates, Geophys. J. R. Astron. Soc., 62, 195-219, 1980.

Pollack, H. N., and D. S. Chapman, On the regional variations of heat flow, geotherms, and lithospheric thickness, Tectonophysics., 38, 279-296, 1977.

Sclater, J. G., C. Jaupart, and D. Galson, The heat flow through oceanic and continental crust and the heat loss of the earth, Rev. Geophys. Space Phys., 18, 264-311, 1980.

Sclater, J. G., B. Parsons, and C. Jaupart, Oceans and continents: Similarities and differences in the mechanisms of heat loss, $J$. Geophys. Res., 86, 11,535-11,552, 1981.

Toksóz, M. N., J. W. Minear, and B. R. Julian, Temperature field and geophysical effects of a downgoing slab, J. Geophys. Res., 76, 1113-1138, 1971.

W. M. Kaula, Department of Earth and Space Sciences, University of California, Los Angeles, CA 90024.

(Received April 2, 1982;

revised June 7, 1983;

accepted June 15, 1983.) 